\title{
In vivo behavior of untreated and compressed concentrated growth factors as biomaterials in rabbits
}

\author{
Takeshi TONE${ }^{1,2}$, Yoshinaka SHIMIZU ${ }^{1}$, Haruka SAITO², Takumi SATO², Hidetoshi ITO'1, Yukyo TAKADA ${ }^{3}$, \\ Masatoshi TAKAHASHI ${ }^{3}$, Kenji ODASHIMA², Mariko OIKAWA ${ }^{1}$, Tetsu TAKAHASHI ${ }^{2}$ and Hiroyuki KUMAMOTO ${ }^{1}$ \\ ${ }^{1}$ Department of Oral Pathology, Graduate School of Dentistry, Tohoku University, 4-1 Seiryo-machi, Aoba-ku, Sendai, Miyagi 980-8575, Japan \\ ${ }^{2}$ Department of Oral and Maxillofacial Surgery, Graduate School of Dentistry, Tohoku University, 4-1 Seiryo-machi, Aoba-ku, Sendai, Miyagi 980- \\ 8575, Japan \\ ${ }^{3}$ Department of Dental Biomaterials, Graduate School of Dentistry, Tohoku University, 4-1 Seiryo-machi, Aoba-ku, Sendai, Miyagi 980-8575, Japan \\ Corresponding author, Yoshinaka SHIMIZU; E-mail: shimizu@dent.tohoku.ac.jp
}

To characterize concentrated growth factors (CGFs) in vivo, we examined the degradation of implanted CGF in rabbits. Untreated CGF (U-CGF) and compressed CGF (C-CGF) were subcutaneously implanted into the dorsum. Histological analyses showed that the U-CGF and C-CGF induced very few inflammatory cells and that the U-CGF and C-CGF were subsequently degraded with dendritic invasion of granulation tissue. The C-CGF histopathologically remained for longer term than the U-CGF. Aggregated CD31+ and RAM11+ cells appeared in and around the implanted CGF. The number of macrophages and blood vessels in the CGF-implanted groups was greater than that in the sham group. There were more blood vessels in the U-CGF group than that in the C-CGF and sham group. We showed that CGF was degraded by macrophages in 4 weeks and enhanced angiogenesis with dendritically branching new capillaries. Therefore, the U-CGF and C-CGF can be clinically applied as a biomaterial inducing angiogenesis.

Keywords: Concentrated growth factor, Tissue regeneration, Wound healing

\section{INTRODUCTION}

Biomaterials, which are required for the practice of current regenerative medicine, have evolved to nextgeneration biomaterials that are bioresorbable and produce bioactive components in living tissues. Platelet concentrates are one of the biomaterials that have been recently developed and designed to activate genes that stimulate tissue regeneration ${ }^{1)}$. As future biomaterials, platelet concentrates are applied to tissue regeneration because of their bioresorbability and biocompatibility.

Platelet-rich plasma (PRP) known as a firstgeneration platelet concentrate containing plasma enriched with growth factors, is utilized for skin wounds treatment and bone reconstruction therapy ${ }^{2)}$. However, PRP has a limited effect on bone regeneration ${ }^{3,4)}$, and its preparation requires extended centrifugation and the use of anticoagulant ${ }^{5}$. Similar to PRP, plateletrich fibrin (PRF) and concentrated growth factor (CGF) are platelet concentrates produced from autologous blood and categorized as second-generation platelet concentrates $^{6)}$. CGF and PRF are produced similarly by single centrifugation of collected blood to avoid the loss of fibrinogen ${ }^{7}$. The difference in the preparation process between CGF and PRF is the speed of centrifugation; the preparation process of $\mathrm{CGF}$ can produce larger and denser growth factors than that of PRF.

Platelets contain a variety of growth factors, including platelet-derived growth factor (PDGF), transforming growth factor- $\beta$ (TGF- $\beta$ ), vascular

Color figures can be viewed in the online issue, which is available at J-STAGE.

Received Sep 4, 2019: Accepted Jan 6, 2020

doi:10.4012/dmj.2018-285 JOI JST.JSTAGE/dmj/2018-285 endothelial growth factor, insulin-like growth factor, ealing and tissue regeneration and maturation ${ }^{8-10)}$. The results of an animal experiment suggested that $\mathrm{CGF}$ containing TGF- $\beta$ and $\mathrm{VEGF}^{7}$ might increase the rate of bone regeneration ${ }^{11)}$, and a clinical study has shown successful flapless transcrestal sinus augmentation using $\mathrm{CGF}^{122}$. In addition, the single application of PRF may not enhance bone healing in extraction sockets ${ }^{13)}$; however, a combination of platelet concentrates and hydroxyapatite or $\beta$-TCP was shown to enhance bone regeneration ${ }^{10,14,15)}$

Currently, CGF is applied to repairing defective tissue as a biomaterial in a membrane- or block-form material covering the tissue. In actual clinical practice for tissue regeneration, platelet concentrates used for tissue regeneration should fulfill the following: be biodegradable, properly maintained, and readily replaced with the original tissue. However, there is very little information describing the behavior, specifically the biodegradation of CGF in vivo. Therefore, to evaluate the efficacy of CGF on tissue regeneration, it is necessary to determine the biodegradation of CGF membrane in vivo and the reaction of CGF to its surrounding tissue. In the present study, we histopathologically and mechanically examined the characteristics of CGF that was subcutaneously implanted into rabbits.

\section{MATERIALS AND METHODS}

CGF preparation

The Committee on the Ethics of Animal Experiments of Tohoku University reviewed the experimental protocols, 
and the experiments were carried out in accordance with the Animal Experiment Guidelines of Tohoku University (2015DnA-016). Approximately $10 \mathrm{~mL}$ of venous blood was taken from the ear veins of rabbits (Fig. 1A) without anticoagulants, directly transferred into a sterile $10-\mathrm{mL}$ tube, and immediately centrifuged in a special centrifuge device (Medifuge, Slifadent Sofia, Italy) for $13 \mathrm{~min}$. The centrifugation program was as follows: 2,700 rpm for $2 \mathrm{~min}, 2,400 \mathrm{rpm}$ for $4 \mathrm{~min}, 2,700$ rpm for $4 \mathrm{~min}$, and 3,000 rpm for $3 \mathrm{~min}^{5)}$ (Fig. 1B). The layer of CGF (approximately $2.5 \mathrm{mg}$ ) was separated from the centrifuged blood sample and divided into two equal volumes. One sample was used without treatment (untreated CGF; U-CGF, Fig. 1C), and the other sample was wrapped with sterile gauze and compressed into a membrane (compressed CGF; C-CGF, Fig. 1D). The C-CGF should possess a certain degree of strength to provide the defective tissue with a constant strength. The property, including the strength, of C-CGF varies depending on compression method. To investigate the method that can standardize the strength of C-CGF, tensile test with $\mathrm{CGF}$ membranes was conducted. The C-CGF was pressed by sterile gauze and acrylic plates (Fig. 1E). The membranes were cut into the sizes of 7 $\mathrm{mm}$ (width) $\times 11 \mathrm{~mm}$ (length). Two knots were tied on each membrane using a nylon surgical suture (USP \#5-0). Each knot was placed $3 \mathrm{~mm}$ from the midpoint of the left or right edge of the membrane. The membranes were soaked in saline solution for $2 \mathrm{~h}$. Tensile tests were conducted using a universal testing machine (AGS$5 \mathrm{kNG}$, Shimadzu, Kyoto, Japan), at a crosshead speed of $5 \mathrm{~mm} / \mathrm{min}$ at room temperature. The maximum load $(\mathrm{N})$ and total elongation $(\mathrm{mm})$ were measured for each

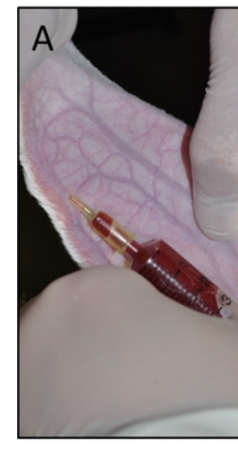

E

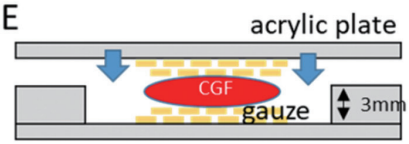

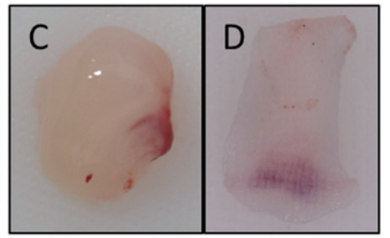

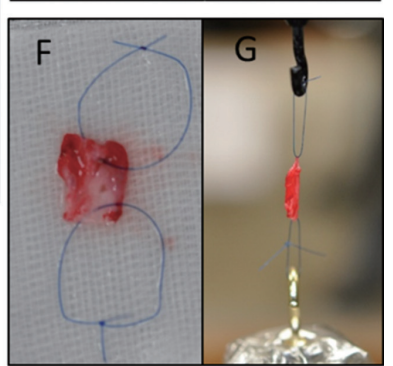

Fig. 1 Preparation of CGF.

(A) Drawing blood from the ear vein of rabbits, (B) Venous blood after centrifugation. Macroscopic findings of $\mathrm{CGF}$, (C) Untreated CGF, (D) Compressed CGF, (E) Drawing press device for CGF, Each two sheets of gauzes up and down of CGF, (F) (G) Tensiled CGF membrane, *: CGF membrane (Figs. 1F and G).

\section{Surgical procedures}

Six white Japanese male rabbits weighing an average of $3 \mathrm{~kg}$ were used in this experiment. Surgery was performed in rabbits under inhalation anesthesia with isoflurane (Wako Pure Chemical Industries, Osaka, Japan). The back of each rabbit was shaved and sterilized with Isodine (Prepodyne, Santen Pharmaceutical, Osaka, Japan). Next, local anesthesia with $2 \%$ lidocaine containing dilute epinephrine 1:100,000 (Xylocaine, Dentsply International, Tokyo, Japan) was given to the dorsal region. Total of three craniocaudal incisions (approximately 20-mm long) were made by blunt dissection $2 \mathrm{~cm}$ lateral to the midline of the dorsal region, creating space between the cutaneous muscle and fat layers to implant CGF (Fig. 2A). The U-CGF (the U-CGF group) and C-CGF (C-CGF group) were implanted into the second and third incisions, respectively (Fig. 2B). The subcutaneous space of the third incision was unfilled as a sham surgery (the sham group). The dissected skin was sutured with a $4 / 0$ nylon thread. The implantation of the $\mathrm{U}-\mathrm{CGF}$ and C-CGF and the sham surgery was performed in a straight line from the head to tail on one side of the back (Fig. 2C). The same surgical procedures mentioned above were performed on the opposite side of the back in the following week. Six rabbits were slaughtered at an overdose of intravascular injection of local anesthetic (Somnopentyl, Kyoritsu Seiyaku, Tokyo, Japan) 1, 2, 3, and 4 weeks after implantation (week 1, 2, 3, and 4).

\section{Histological preparation}

The slaughtered rabbits were given an intracardial
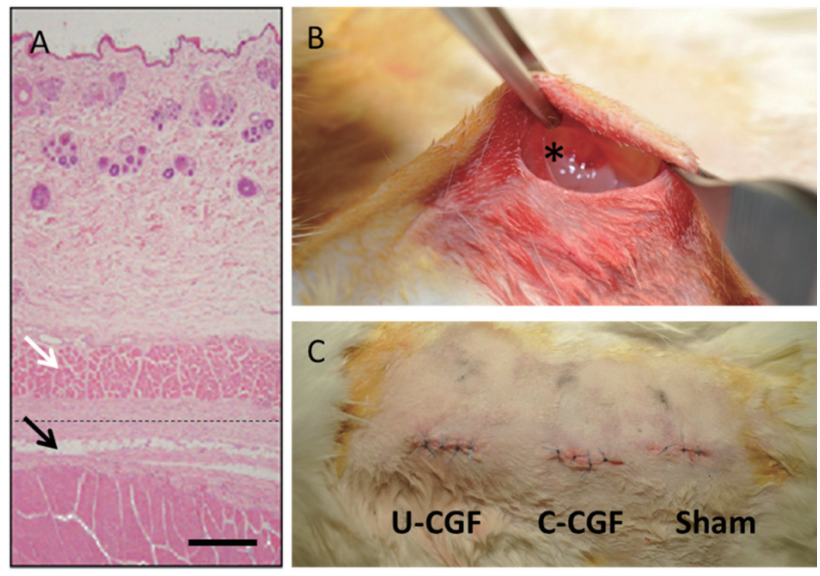

Fig. 2 Surgical procedure.

(A) Histological feature of the dorsal skin, Dotted line: implantation of CGF (between the cutaneous muscle (white arrow) and the fat layer (black arrow), (B) Macroscopic feature of implantation of CGF, (C) Three implantation sites in the dorsal region (U-CGF; Untreated CGF, C-CGF; Compressed CGF, Sham; Sham operation), *: CGF. Scale bar $=1 \mathrm{~mm}$ 
injection of $10 \%$ buffered formalin for irrigation of the entire body of the rabbits. Then, the excised implantation area was re-fixed at $3^{\circ} \mathrm{C}$ for approximately 1 week. The implanted areas including both the U-CGF group and C-CGF group were extracted and embedded separately in paraffin, and serial sections ( $4 \mu \mathrm{m}$-thick) of the paraffin blocks were stained with hematoxylin and eosin (HE), Elastica-Masson (E\&M), or phosphotungstic acidhematoxylin (PTAH). The specimens were observed and examined using a bright-field microscope (Nikon Eclipse Ci, Nikon, Tokyo, Japan).

\section{Immunohistochemical staining}

Sections (4- $\mu \mathrm{m}$ thick) of formalin-fixed paraffin embedded tissues were incubated with antibodies against CD31 and RAM11 to identify infiltrating cells. Immunohistochemical staining was performed following the avidin-biotin-peroxidase system with the monoclonal antibodies as follows: anti-CD31 (CD31/PECAM-1, clone JC/70A, Novus Biologicals, Fontana, CA, USA) and antiRAM-11 (specific for rabbit macrophages, Dako, Grostlap, Denmark). The slides were deparaffinized, hydrated, and treated with $3 \%$ hydrogen peroxide with methanol for $5 \mathrm{~min}$ to block endogenous peroxidase activity. The slides were rinsed with water and then a buffered solution. The slides were incubated overnight at room temperature with anti-CD31 and -RAM11 diluted 1:20 and 1:50 in anitibody diluent (Dako REAL ${ }^{\mathrm{TM}}$ Antibody Dilient, Dako), respectively. The slides were treated with the Dako EnVision+System for $60 \mathrm{~min}$, followed by incubation with $1 \mathrm{mg} / \mathrm{mL}$ DAB solution. The slides were counterstained with Mayer's hematoxylin and washed with phosphate-buffered saline before proceeding to the next reaction step.

\section{Histomorphometrical analysis}

The number of CD31-positive vessels and RAM11positive macrophages in areas surrounding implanted CGF was counted in three low-power fields $(\times 100)$ at week 1 to 4 the above noted microscope (Nikon Eclipse $\mathrm{Ci}$ ). The mean and standard error (SE) were calculated. All data was compared using the two-way ANOVA method and the Bonferroni correction. A $p$-value of $<0.05$ was considered statistically significant.

\section{RESULTS}

\section{Tensile test of $C$ - $C G F$}

Eleven specimens were examined by the tensile test. The results were shown by the strength and strain: $0.32 \pm 0.007 \mathrm{~N}$ (maximum value: $0.48 \mathrm{~N}$, minimum value: $0.18 \mathrm{~N}$ ), and $2.27 \pm 0.24 \mathrm{~mm}$ (maximum value: $3.01 \mathrm{~mm}$, minimum value: $1.55 \mathrm{~mm}$ ), respectively.

\section{Macroscopic findings}

None of the rabbits exhibited complications or pathological symptoms (redness and infected lesion) after surgery. In the cross-sections of the implanted area, white nodules were observed at week 1 and 2 . There was no residual CGF in two of the three rabbits at week 3 and none in

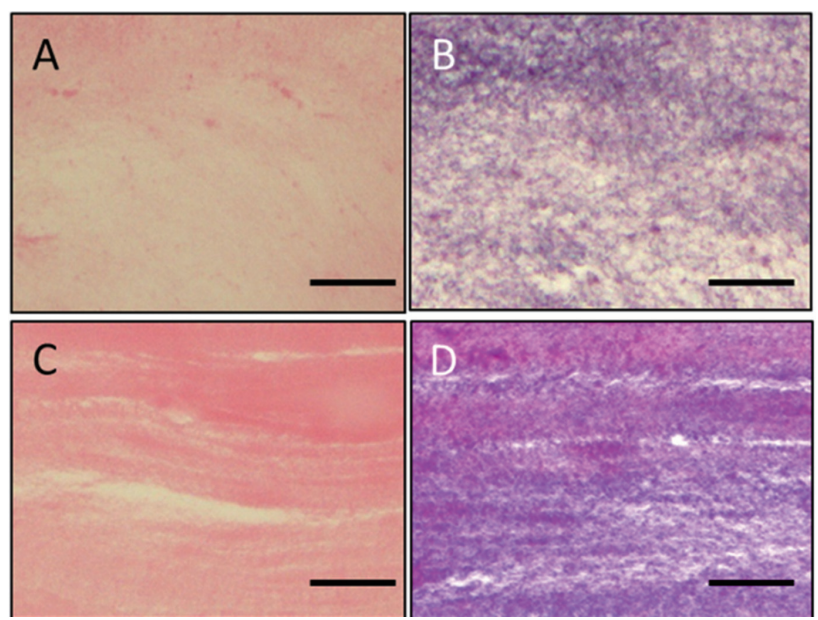

Fig. 3 Histological analysis of CGF.

(A) Untreated CGF, H\&E, (B) Untreated CGF, PTAH, (C) Compressed CGF, H\&E, (D) Compressed $\mathrm{CGF}, \mathrm{PTAH}$, scale bar $=50 \mu \mathrm{m}$

the third rabbit after week 4 .

\section{Histopathological findings}

Figures 3A-D shows the histological findings in the U-CGF (group) and C-CGF (group). HE staining showed eosinophil-infiltrated tissues (fibrin) in both the U-CGF and C-CGF groups (Figs. 3A and C), and PTAH staining showed blue or purple- colored U-CGF and C-CGF (Figs. $3 \mathrm{~B}$ and $\mathrm{D})$. The $\mathrm{C}-\mathrm{CGF}$ was more densely stained with PTAH than the U-CGF (Figs. 3B and D).

The implanted area comprised the epidermis, dermis, thin cutaneous muscle, adipose tissue, and the muscle layer. The $\mathrm{C}-\mathrm{CGF}$ and $\mathrm{U}-\mathrm{CGF}$ in vivo showed the eosinophil-infiltrated tissue in HE staining (Figs. $4 \mathrm{~A}$ and $\mathrm{F}$ ). In EM staining, the CGF showed brown aggregated tissue adjacent to collagen fibers (blue-green) in the dermis and muscle fiber bundles (dark red-brown) (Figs. 4B and G).

Figures 4B-E and 4G-J show histological findings in E\&M staining at week 1, 2, 3 and 4. (The CGF nodule or membrane was implanted beneath the cutaneous muscle.) In the U-CGF and C-CGF groups, certain areas were occupied by $\mathrm{CGF}$, and the dimensions were approximately $9 \times 2 \mathrm{~mm}$ and $7 \times 2 \mathrm{~mm}$ week 1 and 2 , respectively. The implanted areas in both groups decreased 2 and 3 weeks after surgery, and most of the CGF disappeared at week 4 . The implanted areas of both groups were replaced with thin fibrous connective tissue. The implanted C-CGF showed stained more densely than the implanted U-CGF at week 1 (Figs. 4A and $\mathrm{F}$ ). The amount of $\mathrm{CGF}$ in the $\mathrm{C}-\mathrm{CGF}$ group showed high variability over a 3 -week period (Fig. 4I).

At week 1, in the U-CGF and C-CGF groups, PTAHpositive CGF was present in the cutaneous muscle and surrounded by granulation tissue with small numbers of inflammatory cells and collagen fibers (Fig. 5). The CGF exhibited a circumscribed surface with a slit-like space 


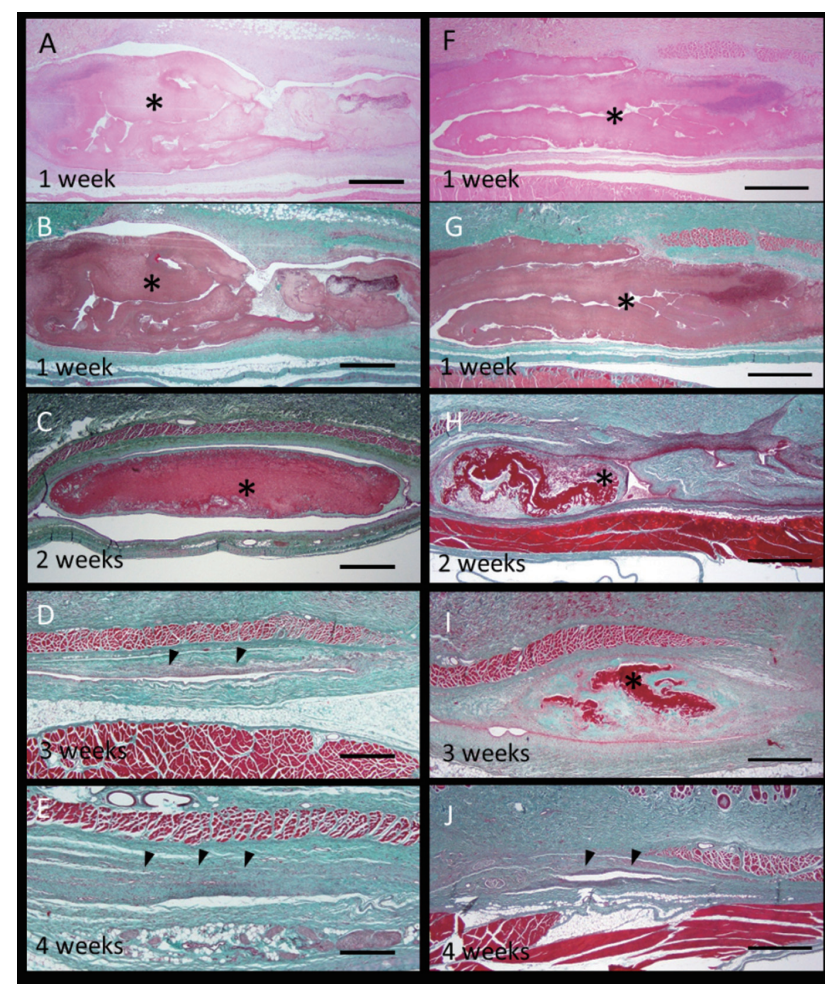

Fig. 4 Histopathological analysis of untreated CGF (A-E) and compressed CGF (F-J) after implantation.

At a week after implantation, there was a little degradation of U-CGF (A, B). At 2 weeks, degradation of U-CGF was progressed (C). Since then 3weeks, there was little CGF in the implanted region (D, E). At a week after implantation, there was a little degradation of C-CGF (F, G). At 2 weeks, degradation of C-CGF was progressed $(\mathrm{H})$. At 3 weeks, C-CGF varying amounts of CGF remained in the implanted region (I). At 4 weeks, there was little $\mathrm{C}$-CGF in the implanted region (J). (A, F) H\&E. (B-E, F-J) E\&M, *: CGF, Scale bar=1 $\mathrm{mm}$, Histopathological analysis of after implantation. (A-D) H\&E, (E-H) E\&M, *: CGF. Arrow: thin fibrous tissue, Scale bar $=1 \mathrm{~mm}$

(Fig. 5A, black arrow head). Rough granulo-connective tissue containing spindle-shaped cells and neutrophils formed within the implanted CGF (Fig. 5).

At week 2, the implanted CGF of both groups were surrounded by adequately vascularized granulation tissues (Fig. 6). The blood vessels and spindle-shaped cells that were contained in the surrounding granulationconnective tissues dendritically and reticulately invaded the CGF.

At week 3, the PTAH-positive CGF was still observed in the implanted area, with its various amount in the C-CGF group (Fig. 7). Fibrous connective tissues with abundant collagen fibers were observed surrounding the CGF. The residual CGF was degraded dendritically by spindle-shaped cells and connective tissues. In the U-CGF group, the CGF was hardly observed in the
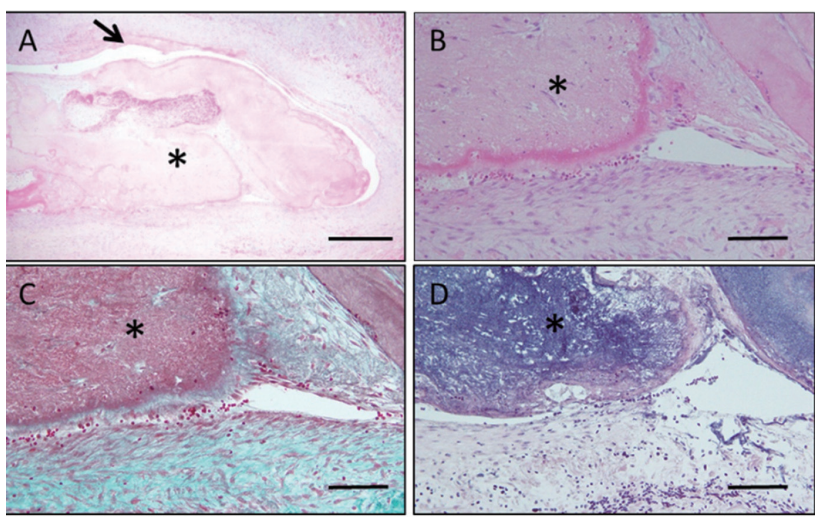

Fig. 5 Untreated CGF after 1 week of implantation.

CGF exhibited a circumscribed surface with a part of slit-like space (A, black arrow). Loose granuloconnective tissue containing spindle-shaped cells and neutrophils formed within the CGF implants (B-D), (A, B) H\&E, (C) E\&M, (D) PTAH, White arrow: loose granulo-connective tissue, *: CGF, Scale bar $=500 \mu \mathrm{m}(\mathrm{A}), 100 \mu \mathrm{m}(\mathrm{B}, \mathrm{C}, \mathrm{D})$

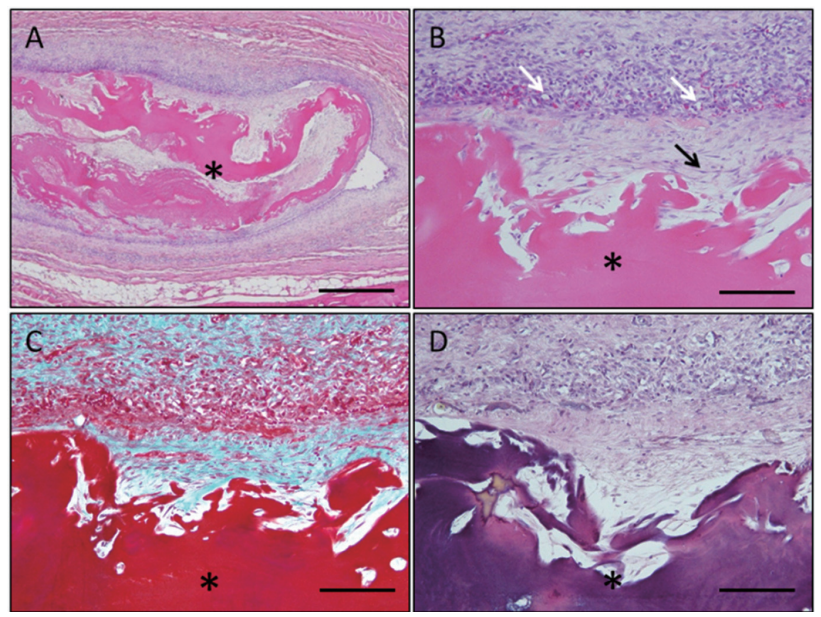

Fig. 6 Compressed CGF after 2 weeks of implantation. The blood vessels (white arrow) and spindle-shaped cells (black arrow) contained in the surrounding granulation-connective tissue revealed dendritic and mesh-like invasion of CGF (A-D). (A, B) H\&E, (C) E\&M, (D) PTAH, *: CGF, Scale bar=1 mm (A), $200 \mu \mathrm{m}$ (B, C, D)

implanted area.

At week 4, there was little residual implanted CGF in the U-CGF and C-CGF groups (Fig. 8), and fibrous connective tissues with collagen fibers were observed in the implanted area. There were no differences in the degradation of $\mathrm{CGF}$ and tissue reactions between the U-CGF and C-CGF groups.

In the sham surgery group, the slight infiltration of inflammatory cells and blood components were observed in the implanted area at week 1 and 2 (Fig. 9), and fibrous connective tissues were observed at week 3 and 

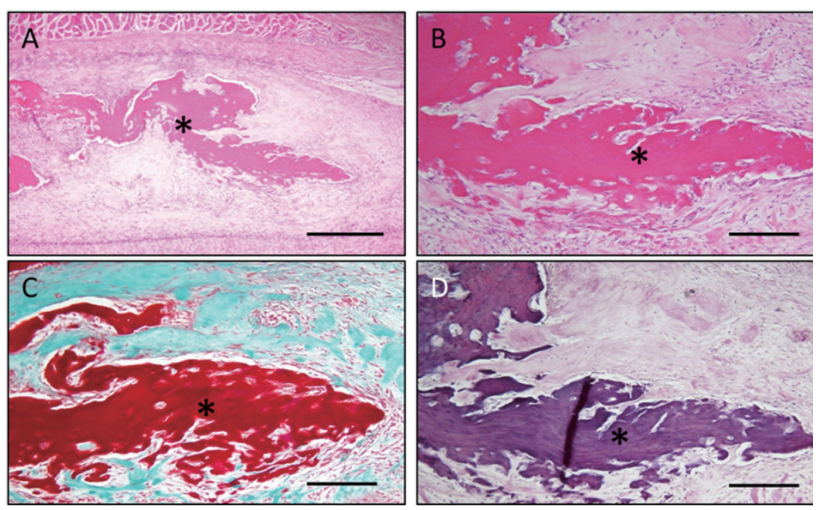

Fig. 7 Compressed CGF after 3 weeks of implantation.

The PTAH-positive CGF varying amounts of CGF remained in the implanted region in the $\mathrm{C}$-CGF group (A-D). (A, B) H\&E, (C) E\&M, (D) PTAH, *: CGF, Scale bar=1 mm (A), $200 \mu \mathrm{m}(\mathrm{B}, \mathrm{C}, \mathrm{D})$
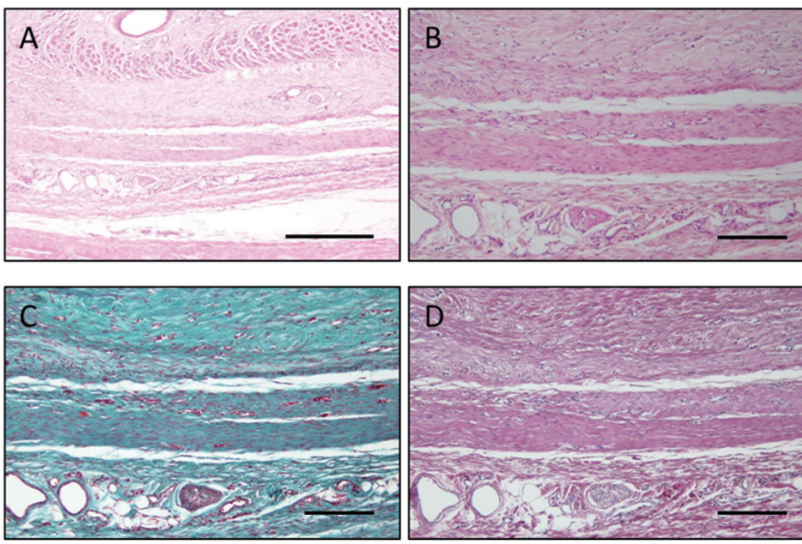

Fig. 8 Untreated CGF 4 weeks after implantation.

There was little residual implanted CGF, and implanted fibrous connective tissue with collagen fibers was present (A-D). (A, B) H\&E, (C) E\&M, (D) PTAH, Scale bar=1 mm (A), $200 \mu \mathrm{m}(\mathrm{B}, \mathrm{C}, \mathrm{D})$

4. These histological findings were consistent with those of the U-CGF and C-CGF groups.

\section{Immunohistochemical findings and histomorphometry}

The CD-31-positive vessels presented with brown ringlike and branched linear structures. The regenerated vessels were observed around or inside the implanted CGF (Fig. 10A-C).

The number of vessels in the sham group did not significantly change up to week 4 . The number of vessels in the U-CGF group was gradually increased until week 3 , with the peak at week 3 in the U-CGF group, and then rapidly decreased at week 4 . The number of vessels at week 4 was significantly less than that at week 1 and $2(p<0.05)$. The number of vessels in the C-CGF group was gradually increased up to week 4 ; at week 3 , it was significantly greater than that at week $1(p<0.05)$. The number of vessels in the U-CGF group was significantly

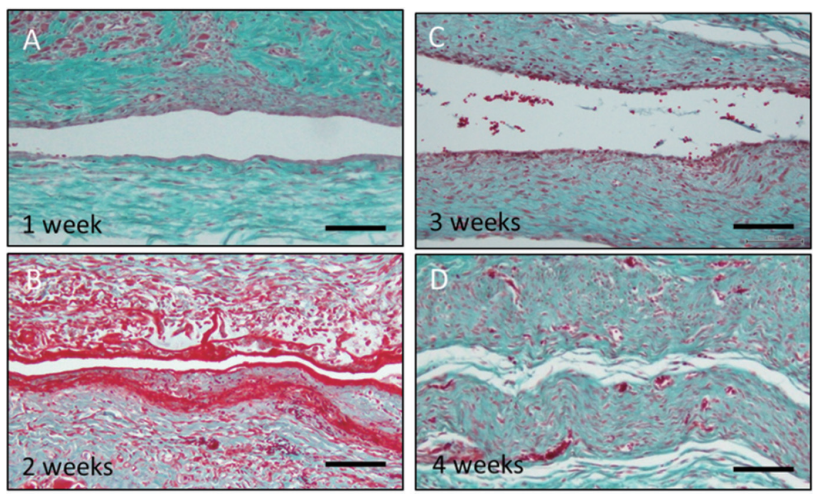

Fig. 9 Histopathological analysis of tissue after sham operations.

The slight infiltration of inflammatory cells and blood components were observed in the surgical area at 1 and 2 weeks (A, B), and fibrous connective tissues were observed after 3 and 4 weeks (C, D). E\&M, Scale bar $=100 \mu \mathrm{m}$
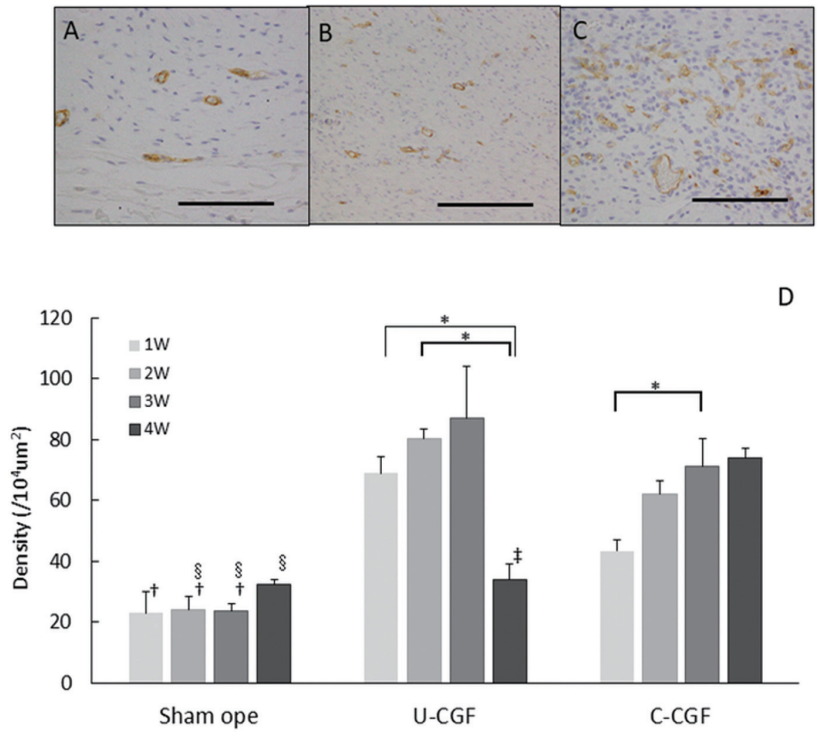

Fig. 10 Immunohistochemical analysis.

The CD-31-positive vessels at 4 weeks were observed in sham ope (A) and around the implanted untreated CGF (B) and compressed CGF (C). Scale bar=100 $\mu \mathrm{m}$. D; Number of CD31positive vessels. ${ }^{*} p<0.05$. $\dagger$ : Sham ope $v s$. U-CGF, §: Sham ope vs. C-CGF, †: U-CGF vs. C-CGF. All symbols $p<0.05$

greater than that in the sham group at week 1,2 and $3(p<0.05)$, and at week 4 the number of vessels was almost the same in the C-CGF and U-CGF groups. The number of vessels in the C-CGF group was significantly greater than that in the sham group at week 2,3 and 4 $(p<0.05)$. In the C-CGF group, it was also significantly greater than that in the U-CGF group at week $4(p<0.05)$ (Fig. 10D). 

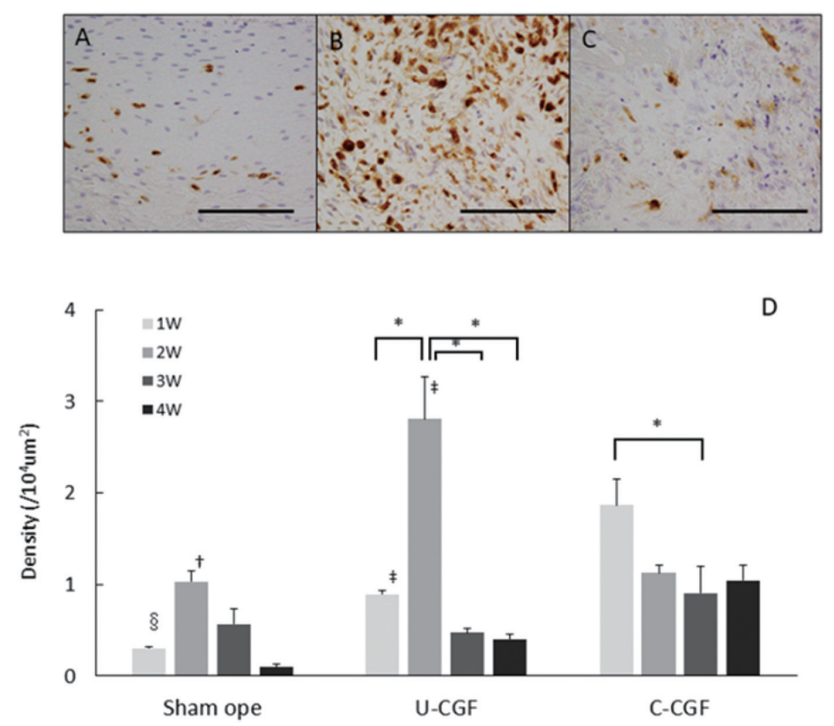

Fig. 11 Immunohistochemical analysis.

The RAM11-positive macrophages at 2 weeks were observed in sham ope (A) and around the implanted untreated CGF (B) and compressed CGF (C). Scale bar=100 $\mu$ m. D; Density of RAM11positive macrophages.

${ }^{*} p<0.05$, $\dagger$ : Sham ope vs. U-CGF, $\S$ : Sham ope vs. C-CGF, †: U-CGF vs. C-CGF, All symbols $p<0.05$

The RAM11-positive macrophages were detected as brown round cells. The macrophages were observed around or inside the implanted CGF (Fig. 11A-C). The density of macrophages peaked at week 2 in the sham and U-CGF groups and then decreased gradually. The density of the macrophages at week 2 was significantly higher than that at week 1,3 and 4 in the U-CGF group $(p<0.05)$. The density of the macrophages showed a peak at week 1 in the C-CGF group and then decreased gradually until week 3 when the density of the macrophages was significantly lower than that at week $1(p<0.05)$. The density of the macrophages in the C-CGF group was significantly higher than that in the sham group and U-CGF group at week $1(p<0.05)$. The density of the macrophages in the U-CGF group was significantly higher than that in the sham group and C-CGF group at week $2(p<0.05)$ (Fig. 11D).

\section{DISCUSSION}

CGF is a platelet-rich fibrin biomaterial ${ }^{5)}$ containing a variety of growth factors. The polymerization of CGF during centrifugation generates a three-dimensional fibrin network ${ }^{5}$. Therefore, the degradation of fibrin and platelets precedes the biodegradation of CGF. Fibrin is degraded by plasmin, which is converted into active plasmin by tissue plasminogen activator, urokinase plasminogen activator, and factor XIII. Our present results indicate that the degradation of CGF was accelerated by the infiltration of macrophages and vessels secreting these plasminogen activators around the implanted CGF.

Lundquist et al. concluded that PRF sustains the release of growth factors that are important for wound healing and protects them against proteolysis ${ }^{16)}$. The duration of activity of the growth factors derived from PRF will be closely related to their degradation rate of a platelet concentrate; however, there are few reports on the degradation rates of a platelet concentrate. Sohn et al. reported that $\mathrm{PRP}, \mathrm{PRF}$, and CGF were observed in wounds for 5-30 days ${ }^{17)}$. For example, according to Soydan et al., PRP persisted for 7 days, while PRF released growth factors during the first 7 days ${ }^{18)}$, or 28 days $^{19)}$, and exerted greater effects of growth factors for longer times ${ }^{20,21)}$. He et al. reported that PRF gradually released growth factors, leading its peak after 14 days $^{21)}$. Our present results showed that when CGF was subcutaneously implanted, it degraded earlier than 1421 days after implantation. Considering the above, these data indicate how long the regenerative effect of growth factors released by implanted CGF lasts.

PRF membrane and/or CGF membrane is occasionally used for clinical practices. To describe this, covering the exposed bone with PRF membrane is effective to close the defects of the jaw and to stimulate gingival healing because PRF acts as a barrier membrane $^{20)}$. This PRF membrane consists of a histologically well-developed mesh of fibrin matrix ${ }^{22)}$. Kobayashi et al. ${ }^{23)}$ reported that to compress PRF affected the plasma content, 3-dimensional fibrin network, number of platelets, and amount of growth factors while minimizing the loss of bioactive factors. The degradation speed of fibrin may be largely determined by fibrin structure, density, number of branch points, porosity, and number of blood vessels in the surrounding tissue. We confirmed that our pressing method enabled the C-CGF to possess mostly consistent mechanical properties. In particular, the stress-strain and compressive-strength of the C-CGF may allow the C-CGF as an artificial membrane ${ }^{24,25)}$ to be clinically applied.

In the comparison between the U-CGF group and C-CGF group, there was no apparent difference in their degradation speed. However, the induction of blood vessels and macrophages infiltration in the U-CGF group was earlier and stronger than that in the C-CGF group. This may indicate differences in the following points between the U-CGF group and the C-CGF group: changes in the three-dimensional structures of the U-CGF, reactions of surrounding tissues caused by the loss of growth factors, and their losing speed. Delaying macrophage infiltration with $\mathrm{C}-\mathrm{CGF}$ can benefit clinical practice because a membrane form of C-CGF remains longer in lesions to prevent other competing tissues from invading. Unlike C-CGF, U-CGF may be useful in different conditions such as a tooth-extraction socket, etc.; As rapid vascular loss was seen at week 4, U-CGF may induce fibrosis, leading to early wound healing.

In the present study, the dendritic intrusion of a capillary-like structure into CGF was observed 2 weeks after implantation. This tissue reaction may 
represent thrombus recanalization. The organization and recanalization of thrombus involves the infiltration of fibroblasts and endothelium into the fibrin-rich thrombus. Thus, CGF, which contains a fibrin matrix and angiogenic factors ${ }^{7}$ may generate the same tissue reaction and cause angiogenesis. Angiogenesis plays key roles in normal development, wound healing, and organ regeneration $^{26)}$. In the early phase of wound healing, angiogenesis contributes to repairing the injured area ${ }^{27)}$ by forming new blood vessels. PRP extract also contains a number of angiogenic growth factors in a certain physiological ratio ${ }^{28,29)}$, and CGF can control angiogenesis in the implanted area because its angiogenic factors are similar to those of PRP and PRF; CGF and PRFderived fibrin structures can allow the long-term release of growth factors from platelets ${ }^{30)}$. In addition, CGF may effectively prevent the proteolysis of the growth factors because of its higher tensile strength and viscosity. The establishment of stable and functional blood vessel networks requires multiple angiogenic growth factors rather than one single factor ${ }^{28}$. Identifying and quantifying the growth factors in CGF is necessary for further improvements in the use of CGF for treating wounds.

Activated platelets secrete various pro- and antiinflammatory factors as well as growth factors that recruit inflammatory immune cells ${ }^{31)}$. These recruited immune cells support angiogenesis during organ development and tissue remodeling ${ }^{32}$. Although optimal vascular formation plays important roles in wound healing, severe inflammation and edema lead to the delay of healing and cause scar tissue formation. He et al. ${ }^{21)}$ stated that TGF- $\beta 1$ and PDGF present in PRF induce the synthesis of collagen and extracellular matrix and the excessive synthesis of collagen fibers leads to scar tissue formation. However, optimal collagen synthesis in the early phase of wound healing process is necessary for successful wound healing. In this study, although reduced blood vessels were observed, the U-CGF and C-CGF group did not show severe inflammation or scar formation around the implanted CGF. Thus, the U- CGF and C-CGF group are considered to be associated with preferable wound healing.

The present study showed that subcutaneously implanted CGF recruited inflammatory cells into the CGF matrix and induced angiogenesis. These findings may lead to novel regenerative therapies using tissue engineering as well as improved treatment of diseases associated with angiogenesis. PRP and PRF are used for many types of regenerative therapies ${ }^{33,34)}$ and can be used as a sole biomaterial or in combination with different constituents $^{35,36)}$. CGF possesses the same biological and mechanistic properties as PRF and may serve as an ideal scaffold for tissue regeneration because of its angiogenic and anti-inflammatory characteristics.

\section{CONCLUSION}

The U-CGF and C-CGF degraded 3 or 4 weeks after subcutaneous implantation in the incisions in the back of the rabbits. Compressing CGF did not largely affect its speed of degradation in vivo. During degradation, abundant blood vessels and macrophages appeared around and inside the U-CGF and C-CGF. However, the decreasing speed of vessels was significantly different between the U-CGF group and the C-CGF group. The difference can be used advantageously for clinical application. Neither of the U-CGF group and C-CGF group induced severe inflammatory reactions nor scar formation. Thus, CGF alone or in combination with other biomaterials can be clinically applied for clinical practices to induce tissue regeneration.

\section{REFERENCES}

1) Hench LL, Polak JM. Third-generation biomedical materials. Science 2002; 295: 1014-1017.

2) Yang HS, Shin J, Bhang SH, Shin JY, Park J, Im GI, et $a l$. Enhanced skin wound healing by a sustained release of growth factors contained in platelet-rich plasma. Exp Mol Med 2011; 43: 622-629.

3) Thorwarth M, Rupprecht S, Falk S, Felszeghy E, Wiltfang J, Schlegel KA. Expression of bone matrix proteins during de novo bone formation using a bovine collagen and plateletrich plasma (prp) —an immunohistochemical analysis. Biomaterials 2005; 26: 2575-2584.

4) Plachokova AS, Nikolidakis D, Mulder J, Jansen JA, Creugers NH. Effect of platelet-rich plasma on bone regeneration in dentistry a systematic review. Clin Oral Implant Res 2008; 19: $539-545$.

5) Honda H, Tamai N, Naka N, Yoshikawa H, Myoui A. Bone tissue engineering with bone marrow-derived stromal cells integrated with concentrated growth factor in Rattus norvegicus calvaria defect model. J Artif Organs 2013; 16: 305-315.

6) Dohan Ehrenfest DM, Rasmusson L, Albrektsson T. Classification of platelet concentrates: from pure platelet-rich plasma (PPRP) to leucocyte- and platelet-rich fibrin (L-PRF). Trends Biotechnol 2009; 27: 158-167.

7) Rodella LF, Favero G, Boninsegna R, Buffoli B, Labanca M, Scarì G, et al. Growth factors, CD34 positive cells, and fibrin network analysis in concentrated growth factors fraction. Microsc Res Tech 2011; 74: 772-777.

8) Yu B, Wang Z. Effect of concentrated growth factors on beagle periodontal ligament stem cells in vitro. Mol Med Rep 2014; 9: 235-242.

9) Kang YH, Jeon SH, Park JY, Chung JH, Choung YH, Choung HW, et al. Platelet-rich fibrin is a bioscaffold and reservoir of growth factors for tissue regeneration. Tissue Eng Part A 2011; 17: 349-359.

10) Nacopoulos C, Dontas I, Lelovas P, Galanos A, Vesalas AM, Raptou $\mathrm{P}$, et al. Enhancement of bone regeneration with the combination of platelet-rich fibrin and synthetic graft. $J$ Craniofac Surg 2014; 25: 2164-2168.

11) Kim TH, Kim SH, Sándor GK, Kim YD. Comparison of platelet-rich plasma (PRP), platelet-rich fibrin (PRF), and concentrated growth factor (CGF) in rabbit-skull defect healing. Arch Oral Biol 2014; 59: 550-558.

12) Kim JM, Sohn DS, Bae MS, Moon JW, Lee JH, Park IS. Flapless transcrestal sinus augmentation using hydrodynamic piezoelectric internal sinus elevation with autologous concentrated growth factors alone. Implant Dent 2014; 23: 168-174.

13) Gürbüzer B, Pikdöken L, Tunali M, Urhan M, Küçükodaci Z, Ercan F. Scintigraphic evaluation of osteoblastic activity in extraction sockets treated with platelet-rich fibrin. J Oral Maxillofac Surg 2010; 68: 980-989. 
14) Yilmaz D, Dogan N, Ozkan A, Sencimen M, Ora BE, Mutlu I. Effect of platelet rich fibrin and beta tricalcium phosphate on bone healing. A histological study in pigs. Acta Cir Bras 2014; 29: 59-65.

15) Bölükbaşı N, Yeniyol S, Tekkesin MS, Altunatmaz K. The use of platelet-rich fibrinin combination with biphasic calcium phosphate in the treatment of bone defects: a histologic and histomorphometric study. Curr Ther Res Clin Exp 2013; 75: 15-21.

16) Lundquist R, Dziegiel MH, Agren MS. Bioactivity and stability of endogenous fibrogenic factors in platelet-rich fibrin. Wound Repair Regen 2008; 16: 356-363.

17) Sohn DS, Moon JW, Moon YS, Park JS, Jung HS. The use of concentrated growth factors (CGF) for sinus augmentation. J Oral Implant 2009; 38: 25-35.

18) Dohan Ehrenfest DM, Bielecki T, Jimbo R, Barbé G, Del Corso M, Inchingolo F, et al. Do the fibrin architecture and leukocyte content influence the growth factor release of platelet concentrates? An evidence-based answer comparing a pure platelet-rich plasma (P-PRP) gel and a leukocyte- and platelet-rich fibrin (L-PRF). Curr Pharm Biotechnol 2012; 13: 1145-1152.

19) Zumstein MA, Berger S, Schober M, Boileau P, Nyffeler RW, Horn M, et al. Leukocyte- and platelet-rich fibrin (L-PRF) for long-term delivery of growth factor in rotator cuff repair: review, preliminary results and future directions. Curr Pharm Biotechnol 2012; 13: 1196-1206.

20) Soydan SS, Uckan S. Management of bisphosphonate-related osteonecrosis of the jaw with a platelet-rich fibrin membrane: technical report. J Oral Maxillofac Surg 2014; 72: 322-326.

21) He L, Lin Y, Hu X, Zhang Y, Wu H. A comparative study of platelet-rich fibrin (PRF) and platelet-rich plasma (PRP) on the effect of proliferation and differentiation of rat osteoblasts in vitro. Oral Surg Oral Med Oral Pathol Oral Radiol Endod 2009; 108: 707-713.

22) Dohan DM, Choukroun J, Diss A, Dohan SL, Dohan AJ, Mouhyi J, et al. Platelet-rich fibrin (PRF): a second-generation platelet concentrate. Part I: technological concepts and evolution. Oral Surg Oral Med Oral Pathol Oral Radiol Endod 2006; 101: e37-44

23) Kobayashi M, Kawase T, Horimizu M, Okuda K, Wolff LF, Yoshie H. A proposed protocol for the standardized preparation of PRF membranes for clinical use. Biologicals 2012; 40: 323-329.

24) Isobe K, Watanebe T, Kawabata H, Kitamura Y, Okudera T,
Okudera H, et al. Mechanical and degradation properties of advanced platelet-rich fibrin (A-PRF), concentrated growth factors (CGF), and platelet-poor plasma-derived fibrin (PPTF). Int J Implant Dent 2017; 17: 81-87.

25) Khorshidi H, Raoofi S, Bagheri R, Banihashemi H. Comparison of the mechanical properties of early leukocyteand platelet-rich fibrin versus PRGF/Endoret membranes. Int J Dent 2016; 2016: 1849207.

26) Carmeliet P, Jain RK. Molecular mechanisms and clinical applications of angiogenesis. Nature 2011; 473: 298-307.

27) Nogami M, Hoshi T, Kinoshita M, Arai T, Takama M, Takahashi I. Vascular endothelial growth factor expression in rat skin incision wound. Med Mol Morphol 2007; 40: 8287.

28) Yancopoulos GD, Davis S, Gale NW, Rudge JS, Wiegand SJ, Holash J. Vascular-specific growth factors and blood vessel formation. Nature 2000; 407: 242-248.

29) Zhou B, Poon MC, Pu WT, Han ZC. Therapeutic neovascularization for peripheral arterial diseases: advances and perspectives. Histol Histopathol 2007; 22: 677-686.

30) Mammoto $T$, Jiang A, Jiang E, Mammoto A. Platelet rich plasma extract promotes angiogenesis through the angiopoietin1-Tie2 pathway. Microvasc Res 2013; 89: 15-24.

31) McNicol A, Israels SJ. Beyond hemostasis: the role of platelets in inflammation, malignancy and infection. Cardiovasc Hematol Disord Drug Targets 2008; 8: 99-117.

32) Nucera S, Biziato D, De Palma M. The interplay between macrophages and angiogenesis in development, tissue injury and regeneration. Int J Dev Biol 2011; 55: 495-503.

33) Kurita J, Miyamoto M, Ishii Y, Aoyama J, Takagi G, Naito $\mathrm{Z}$, et al. Enhanced vascularization by controlled release of platelet-rich plasma impregnated in biodegradable gelatin hydrogel. Ann Thorac Surg 2011; 92: 837-844.

34) Sánchez-González DJ, Méndez-Bolaina E, Trejo-Bahena NI. Platelet-rich plasma peptides: key for regeneration. Int $\mathrm{J}$ Pept 2012: 532519.

35) Yoon JS, Lee SH, Yoon HJ. The influence of platelet-rich fibrin on angiogenesis in guided bone regeneration using xenogenic bone substitutes: A study of rabbit cranial defects. J Craniomaxillofac Surg 2014; 42: 1071-1077.

36) Jang ES, Park JW, Kweon H, Lee KG, Kang SW, Baek DH, et al. Restoration of peri-implant defects in immediate implant installations by Choukroun platelet-rich fibrin and silk fibroin powder combination graft. Oral Surg Oral Med Oral Pathol Oral Radiol Endod 2010; 109: 831-836. 